\title{
Towards an efficient description of strongly correlated electrons with mean-field cost
}

\author{
Katharina Boguslawski, Paweł Tecmer, and Paul W. Ayer:** \\ Department of Chemistry and Chemical Biology, \\ McMaster University, Hamilton, \\ 1280 Main Street West, L8S 4M1, Canada \\ Patrick Bultinck \\ Department of Inorganic and Physical Chemistry, \\ Ghent University, Krijgslaan 281 (S3), 9000 Gent, Belgium
}

\author{
Stijn De Baerdemacker and Dimitri Van Neck $\rceil^{\dagger}$ \\ Center for Molecular Modelling, Ghent University, \\ Technologiepark 903, 9052 Gent, Belgium
}

(Dated: April 17, 2014)

\begin{abstract}
We present an efficient approach to the electron correlation problem that is well-suited for strongly interacting many-body systems, but requires only mean-field-like computational cost. The performance of our approach is illustrated for the one-dimensional Hubbard model with periodic boundary conditions for different chain lengths, and for the non-relativistic quantum chemical Hamiltonian exploring the symmetric dissociation of the $\mathrm{H}_{50}$ hydrogen chain.
\end{abstract}

The accurate description of the electron-electron interaction at the quantum-mechanical level is a key problem in condensed matter physics and quantum chemistry. Since most of the quantum many-body problems are extraordinarily difficult to solve exactly, different approximation schemes emerged [5] 9], among which the density matrix renormalization group (DMRG) algorithm [1012. gained a lot of popularity in both condensed matter physics [11] and quantum chemistry [13,20] over the last decade. Since the DMRG algorithm optimizes a matrix product state wavefunction, it is optimally suited for one-dimensional systems; though DMRG studies on higher-dimensional and compact systems have been reported [14, 17, 19, 21]. Yet, novel theoretical approaches are desirable that can accurately describe strong correlation effects between electrons where the dimension of the Hilbert space exceeds the present-day limit of DMRG or general tensor-network approaches 22 allowing approximately 100 sites or 60 (spatial) orbitals, respectively.

Another promising approach, suitable for larger strongly-correlated electronic systems, uses geminals (two-electron basis functions) as building blocks for the wavefunction [23 30]. One of the simplest practical geminal approaches is the antisymmetric product of 1reference-orbital geminals (AP1roG) 31-33. Unique among geminal methods, AP1roG can be rewritten as a fully general pair-coupled-cluster doubles wavefunc-

\footnotetext{
* ayers@mcmaster.ca

$\dagger$ dimitri.vanneck@ugent.be
}

tion [34, i.e.

$$
\left|\Psi_{\mathrm{AP} 1 \mathrm{roG}}\right\rangle=\exp \left(\sum_{i=1}^{P} \sum_{a=P+1}^{K} c_{i}^{a} a_{a \uparrow}^{\dagger} a_{a \downarrow}^{\dagger} a_{i \downarrow} a_{i \uparrow}\right)\left|\Phi_{0}\right\rangle,
$$

where $a_{p \sigma}^{\dagger}$ and $a_{p \sigma}(\sigma=\downarrow, \uparrow)$ are the fermionic creation and annihilation operators, and $\left|\Phi_{0}\right\rangle$ is some independent-particle wavefunction (usually the HartreeFock determinant). Indices $i$ and $a$ correspond to virtual and occupied sites (orbitals) with respect to $\left|\Phi_{0}\right\rangle, P$ and $K$ denote the number of electron pairs $(P=N / 2$ with $N$ being the total number of electrons) and orbitals, respectively, and $\left\{c_{i}^{a}\right\}$ are the geminal coefficients. This wavefunction ansatz is size-extensive and has mean-field scaling, $\mathcal{O}\left(P^{2}(K-P)^{2}\right)$ for the projected Schrödinger equation approach 31 .

To ensure size-consistency, however, it is necessary to optimize the one-electron basis functions [31, where all non-redundant orbital rotations span the occupiedoccupied, occupied-virtual, and virtual-virtual blocks with respect to the reference Slater determinant $\left|\Phi_{0}\right\rangle$. We have implemented a quadratically convergent algorithm: we minimize the energy with respect to the choice of the one-particle basis functions, subject to the constraint that the projected Schrödinger equations for the geminal coefficients hold. Specifically, we use a NewtonRaphson optimizer and a diagonal approximation of the orbital Hessian to obtain the rotated set of orbital expansion coefficients. Our algorithm is analogous to the orbital-optimized coupled cluster approach [35-37. Due to the four-index transformation of the electron repulsion integrals, the computational scaling deteriorates to $\mathcal{O}\left(K^{5}\right)$. The orbital-optimized AP1roG (OO-AP1roG) approach was implemented in a developer version of the 


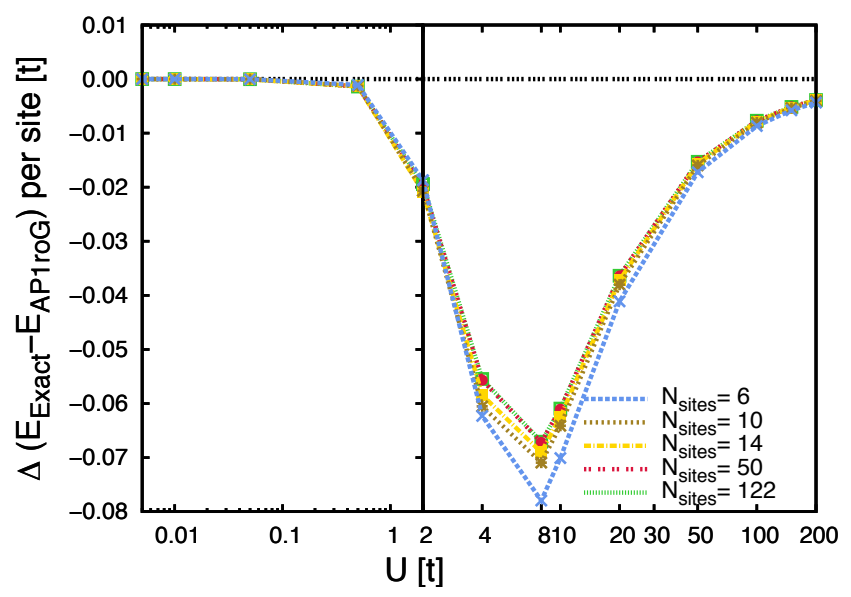

FIG. 1. Deviation of the OO-AP1roG total energies from exact values (blue dashed line) for different strengths of the repulsive on-site interaction for the 1-D Hubbard model (with periodic boundary conditions) for $\mathrm{N}_{\text {sites }}=6,10,14,50,122$. The exact values for small $U(U<0.001 t)$ for $\mathrm{N}_{\text {sites }}=50,122$ could not be converged.

HORTON program package [38.

(a) The half-filled one-dimensional Hubbard Hamiltonian. First, we consider the 1-D Hubbard model Hamiltonian with periodic boundary conditions,

$\hat{H}_{\mathrm{Hub}}=-t \sum_{j, \sigma}\left(a_{(j+1) \sigma}^{\dagger} a_{j \sigma}+a_{j \sigma}^{\dagger} a_{(j+1) \sigma}\right)+U \sum_{j} n_{j \uparrow} n_{j \downarrow}$,

where the first term represents nearest-neighbor hopping and the second term is the repulsive on-site interaction. The operators $a_{j \sigma}^{\dagger}$ and $a_{j \sigma}$ are again the fermionic creation and annihilation operators on a lattice with sites $j=1, \ldots, \mathrm{N}_{\text {sites }}$, and $n_{j \sigma}=a_{j \sigma}^{\dagger} a_{j \sigma}$ is the local number operator.

Figure 1 shows the differences in total energies obtained for OO-AP1roG with respect to reference data obtained from the solution of the Lieb-Wu equations [39. $\left(\mathrm{N}_{\text {sites }}=6,10,14,50,122\right)$. OO-AP1roG can reproduce the exact total energies in the limit of zero and infinite (repulsive) on-site interaction. The largest deviations from the exact solution (up to $0.075 t$ per site) are found for the intermediate region of the on-site interaction, that is, for $2 t<U<50 t$. Figure 2 shows the percentage of the correlation energy captured by OO-AP1roG calculated as $\% \kappa=\frac{E^{\mathrm{OO}-\mathrm{AP} 1 \mathrm{roG}}-E^{\mathrm{HF}}}{E^{\text {exact }}-E^{\mathrm{HF}}} \cdot 100$. In the limit of zero and infinite $U$, the OO-AP1roG model becomes exact; for $U=0$ the wavefunction can be exactly described by a single Slater determinant and thus the correlation energy approaches zero, while for $U \rightarrow \infty$, the quantum state can be represented by the perfect pairing wavefunction. For growing (repulsive) $U$, the percentage of the correlation energy covered by OO-AP1roG increases gradually.

For small values of $U$, the geminal coefficient matrix $\left\{c_{i}^{a}\right\}$ is sparse and thus far from perfect pairing, which is represented by a diagonal geminal coefficient matrix

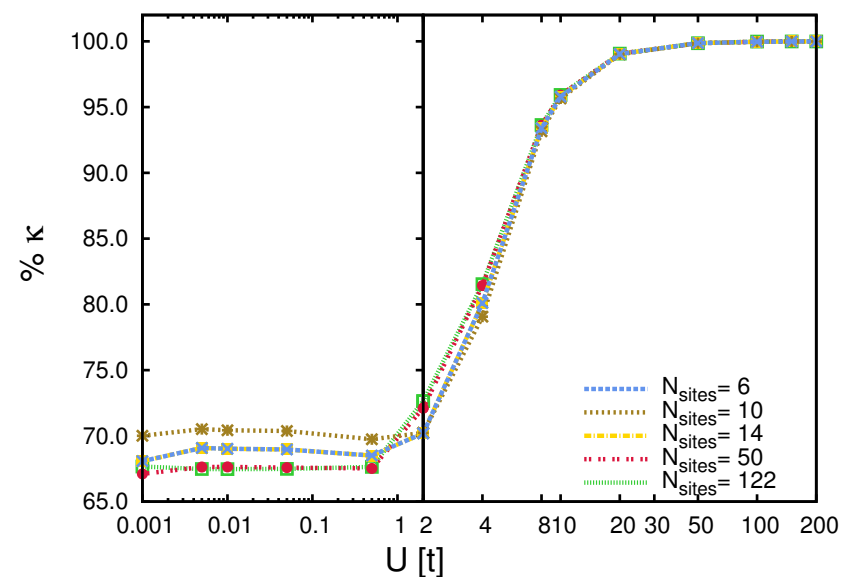

FIG. 2. Percentage of the correlation energy $\% \kappa$ for different strengths of the repulsive on-site interaction in the half-filled 1-D Hubbard model (with periodic boundary conditions) for $\mathrm{N}_{\text {sites }}=6,10,14,50,122$ captured by OO-AP1roG. The exact values for small $U(U<0.001 t)$ for $\mathrm{N}_{\text {sites }}=50,122$ could not be converged.

(see Figure I in the Supplementary Information). In the limit $U \rightarrow 0$, the geminal coefficient matrix correctly approaches the zero matrix indicating that a single Slater determinant is sufficient to describe the quantum state exactly. For increasing $U,\left\{c_{i}^{a}\right\}$ becomes diagonaldominant and adopts a diagonal structure in the limit of $U \rightarrow \infty$. Thus, in the limit of infinite (repulsive) interaction, OO-AP1roG optimizes a perfect-pairing (seniorityzero) wavefunction [40, 41],

$$
\prod_{i=1,3, . .}\left[\left(a_{i, \uparrow}^{\dagger}+a_{i+1, \uparrow}^{\dagger}\right)\left(a_{i, \downarrow}^{\dagger}+a_{i+1, \downarrow}^{\dagger}\right)-\left(a_{i, \uparrow}^{\dagger}-a_{i+1, \uparrow}^{\dagger}\right)\left(a_{i, \downarrow}^{\dagger}-a_{i+1, \downarrow}^{\dagger}\right)\right]|0\rangle
$$

To conclude, OO-AP1roG has mean-field-like scaling, but can recover about $71 \%$ of the correlation energy in the weak interaction regime, about $80 \%$ for intermediate interaction strengths, and approximately $93 \%$ in the case of strong on-site interaction for all chain lengths studied (a numerical comparison is presented in Table I of the Supplementary Information).

Figure 3 shows the single-orbital entropy for different lengths of the 1-D lattice as a function of the repulsive on-site interaction $U$. The single-orbital entropy is the analogue of the one-site entropy, but determined in the natural orbital basis: it is calculated as the von Neumann entropy from a single-orbital density matrix (a manyparticle reduced density matrix of one orbital). It measures the entanglement of one orbital with the remaining $\left(\mathrm{N}_{\text {sites }}-1\right)$ ones [18. In particular, since the optimized orbitals are localized on two neighboring sites, the von Neumann entropy describes the correlation of a pair of sites and the other part of the system. In the following, we will refer to the single-orbital entropy as the pair entanglement $E_{p}$ in accordance with the local entanglement determined for the on-site basis [44. 


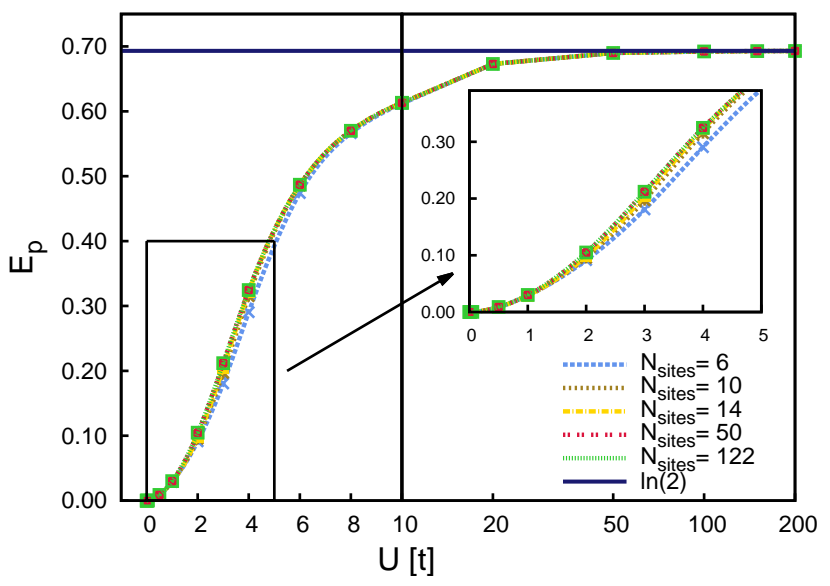

FIG. 3. Pair entanglement $E_{p}$ (single-orbital entropy) for different strengths of the repulsive on-site interaction in the half-filled 1-D Hubbard model (with periodic boundary conditions) for $\mathrm{N}_{\text {sites }}=6,10,14,50,122$ calculated by OO-AP1roG.

The pair entanglement takes its minimum value at $U=0 t$ where the wavefunction can be exactly represented by a single Slater determinant. It is easy to verify that all orbital pairs are uncorrelated in a onedeterminant wavefunction and thus $E_{p}=0$. For increasing on-site interaction, the pair entanglement smoothly accumulates (see Figure 3) and reaches its maximum value of $\ln 2$ in the large $U$ limit (for $U \rightarrow \infty$, the single-orbital density matrix has the diagonal elements $\{0,0,0.5,0.5\})$. Note that OO-AP1roG yields similar pair entanglement profiles for all chain lengths studied and correctly reproduces the small and large $U$ limits.

(b) Symmetric dissociation of the $\mathbf{H}_{50}$ molecule. The non-relativistic quantum chemical Hamiltonian in its second quantized form reads

$\hat{H}=\sum_{p q, \sigma} h_{p q} a_{p \sigma}^{\dagger} a_{q \sigma}+\frac{1}{2} \sum_{p q r s, \sigma \tau}\langle p q \mid r s\rangle a_{p \sigma}^{\dagger} a_{q \tau}^{\dagger} a_{s \tau} a_{r \sigma}+H_{\mathrm{nuc}}$,

where the first term comprises the kinetic energy and nuclear-electron attraction, the second term is the electron-electron interaction, and the third term represents the nuclear-nuclear repulsion energy, respectively. In Eq. (4), indices $p, q, r$ and $s$ run over all one-particle basis functions, while $\sigma$ and $\tau$ denote the electron spin $(\{\uparrow, \downarrow\})$. The Hamiltonian as defined in Eq. (4) was used for the study of the symmetric stretching of the $\mathrm{H}_{50}$ hydrogen chain, which is a commonly-used molecular model for strongly-correlated systems and which remains a challenging problem for conventional quantumchemistry methods 45 48.

In Figure 4, the performance of AP1roG and OOAP1roG is compared to restricted Hartree-Fock (RHF), second-order Møller-Plesset (MP2) perturbation theory, coupled cluster theory with singles, doubles and perturbative triples $(\operatorname{CCSD}(\mathrm{T}))$, and density functional theory using the PBE [50] exchange-correlation functional. As

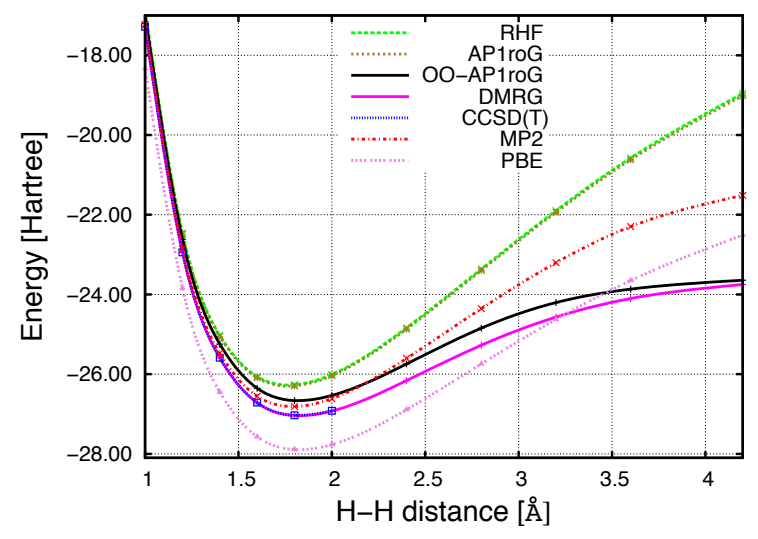

FIG. 4. Symmetric dissociation of $\mathrm{H}_{50}$ chain using the STO6G basis set 49] obtained from different methods. The DMRG reference data are taken from Ref. 45.

TABLE I. Spectroscopic constants: equilibrium bond distance $\left(R_{e}\right)$, potential energy depth $\left(D_{e}\right)$ and harmonic vibrational frequency $\left(\omega_{\mathrm{e}}\right)$ for the ground state of $\mathrm{H}_{50}$ (STO-6G). Differences with respect to the DMRG reference data are listed in parenthesis.

\begin{tabular}{llll}
\hline \hline Method & $\mathrm{R}_{\mathrm{e}}[\AA]$ & $\mathrm{D}_{\mathrm{e}}[\mathrm{eV}]$ & $\omega_{\mathrm{e}}\left[\mathrm{cm}^{-1}\right]$ \\
\hline RHF & $0.940(-0.030)$ & $199.0(+109.3)$ & $25089(+2268)$ \\
AP1roG & $0.941(-0.029)$ & $198.2(+108.5)$ & $23013(+2252)$ \\
MP2 & $0.955(-0.015)$ & $144.1(+54.4)$ & $24568(+1747)$ \\
PBE & $0.971(+0.001)$ & $146.6(+56.9)$ & $23662(+841)$ \\
OO-AP1roG & $0.966(-0.004)$ & $82.2(-7.5)$ & $23013(+192)$ \\
DMRG [4] & 0.970 & 89.7 & 22821 \\
\hline \hline
\end{tabular}

reference, the DMRG potential energy curve determined in Ref. 45] was used, which can be considered as the exactdiagonalization limit. None of the standard quantum chemical methods, like MP2, $\operatorname{CCSD}(\mathrm{T})$ or DFT using the PBE exchange-correlation functional, yield qualitatively correct energy curves for the symmetric stretching of the $\mathrm{H}_{50}$ chain. In particular, the potential energy depth determined from DFT and MP2 is too deep, while $\operatorname{CCSD}(\mathrm{T})$ does not converge for interatomic distances larger than $2.0 \AA$. Note that the lack of size-consistency in AP1roG is cured by orbital optimization in the OOAP1roG approach. The latter yields a potential energy curve that is closest to the DMRG reference data along the whole dissociation pathway and leads to a proper dissociation limit of $\mathrm{H}_{50}$. Moreover, the OO-AP1roG method gives spectroscopic constants (presented in Table (I) that are in excellent agreement with DMRG reference data, outperforming standard quantum-chemistry approaches.

Wavefunctions constructed as antisymmetric products of nonorthogonal geminals, like the AP1roG wavefunction scrutinized here, provide an alternative approach to electronic structure, with mean-field scaling. Because it uses electron pairs as a building block, AP1roG is a suitable way to describe strong correlations dominated by electron pairing. However, in order to ensure sizeconsistency, the single-particle (orbital) basis used to 
construct the electron pairs must be optimized. Our results show that orbital-optimized AP1roG is a robust method all the way from the weakly-correlated to the strongly-correlated limit, in both molecules and periodic systems.

Acknowledgments. K.B. acknowledges the financial support from the Swiss National Science Foundation (P2EZP2 148650). P.T. and P.W.A gratefully acknowledge financial support from the Natural Sciences and Engineering Research Council of Canada. P.B. with
S.D.B. and D.V.N acknowledge financial support from FWO-Flanders and the Research Council of Ghent University. S.D.B is an FWO postdoctoral fellow. We thank Ward Poelmans, Brecht Verstichel and Paul Johnson for results for the Hubbard model obtained by solving the Lieb-Wu equations. We had many helpful discussions on geminals and exactly-solvable models with Peter Limacher and Paul Johnson. B.V., H.V.A., W.P., S.W. and D.V.N. are Members of the QCMM alliance GhentBrussels.
[1] E. Dagotto, Rev. Mod. Phys. 66, 763 (1994).

[2] K. Capelle and V. L. Campo, Phys. Report. 528, 91 (2013).

[3] P.-O. Löwdin, in Adv. Chem. Phys., Vol. I (Wiley \& Sons, Inc, 1958) Chap. Review of different approaches and discussion of some current ideas, pp. 209-321.

[4] R. J. Bartlett and M. Musiał, Rev. Mod. Phys. 79, 291 (2007).

[5] W. Foulkes, L. Mitas, R. Needs, and G. Rajagopal, Rev. Mod. Phys. 73, 33 (2001).

[6] D. I. Lyakh, M. Musial, V. F. Lotrich, and . J. Bartlett, Chem. Rev. 112, 182 (2012).

[7] G. K.-L. Chan and S. Sharma, Annu. Rev. Phys. Chem. 62, 465 (2011).

[8] L. Pollet, Rep. Prog. Phys. 75, 094501 (2012).

[9] R. Rodríguez-Guzmán, C. A. Jiménez-Hoyos, R. Schutski, and G. E. Scuseria, Phys. Rev. B 87, 235129 (2013).

[10] S. R. White, Phys. Rev. Lett. 69, 2863 (1992).

[11] U. Schollwöck, Rev. Mod. Phys. 77, 259 (2005).

[12] O. Legeza, R. M. Noack, J. Sólyom, and L. Tincani, in Computational Many-Particle Physics, Lect. Notes Phys., Vol. 739, edited by H. Fehske, R. Schneider, and A. Weiße (Springer, Berlin/Heidelerg, 2008) pp. 653-664.

[13] K. H. Marti and M. Reiher, Z. Phys. Chem. 224, 583 (2010).

[14] K. Boguslawski, K. H. Marti, O. Legeza, and M. Reiher, J. Chem. Theory Comput. 8, 1970 (2012).

[15] K. Boguslawski, P. Tecmer, O. Legeza, and M. Reiher, J. Phys. Chem. Lett. 3, 3129 (2012).

[16] S. Wouters, P. A. Limacher, D. Van Neck, and P. W. Ayers, J. Chem. Phys. 136, 134110 (2012).

[17] Y. Kurashige, G. K.-L. Chan, and T. Yanai, Nature Chem. 5, 660 (2013).

[18] K. Boguslawski, P. Tecmer, G. Barcza, O. Legeza, and M. Reiher, J. Chem. Theory Comput. 9, 2959 (2013).

[19] P. Tecmer, K. Boguslawski, O. Legeza, and M. Reiher, Phys. Chem. Chem. Phys 16, 719 (2014).

[20] S. Wouters, W. Poelmans, P. W. Ayers, and D. Van Neck, Comput. Phys. Comm. XX, DOI:10.1016/j.cpc.2014.01.019 (2014).

[21] M. C. Chung and I. Peschel, Phys. Rev. B 62, 4191 (2000).

[22] V. Murg, F. Verstraete, O. Legeza, and R. M. Noack, Phys. Rev. B 82, 205105 (2010).

[23] A. C. Hurley, J. Lennard-Jones, and J. A. Pople, Proc. R. Soc. Lond. A 220, 446 (1953).

[24] A. J. Coleman, J. Math. Phys. 6, 1425 (1965).

[25] D. M. Silver, J. Chem. Phys. 50, 5108 (1969).
[26] J. V. Ortiz, B. Weiner, and Y. Ohrn, Int. J. Quantum Chem. S15, 113 (1981).

[27] P. R. Surjan, in Correlation and Localization (Springer, 1999) pp. 63-88.

[28] W. Kutzelnigg, Chem. Phys. 401, 119 (2012).

[29] P. R. Surján, A. Szabados, P. Jeszenszki, and T. Zoboki, J. Math. Chem. 50, 534 (2012).

[30] J. K. Ellis, R. L. Martin, and G. E. Scuseria, J. Chem. Theory Comput. 9, 2857 (2013).

[31] P. A. Limacher, P. W. Ayers, P. A. Johnson, S. De Baerdemacker, D. Van Neck, and P. Bultinck, J. Chem. Theory Comput. 9, 1394 (2013).

[32] P. Limacher, P. Ayers, P. Johnson, S. De Baerdemacker, D. Van Neck, and P. Bultinck, Phys. Chem. Chem. Phys 16, 5061 (2014).

[33] P. A. Limacher, T. D. Kim, P. W. Ayers, P. A. Johnson, S. De Baerdemacker, D. Van Neck, and P. Bultinck, Mol. Phys., 853 (2014).

[34] T. M. Henderson, J. Dukelsky, G. E. Scuseria, A. Signoracci, and T. Duguet, arXiv preprint arXiv:1403.6818 (2014).

[35] T. Helgaker, P. Jørgensen, and J. Olsen, Molecular Electronic-Structure Theory (Wiley, 2000).

[36] G. E. Scuseria and H. F. Schaefer III, Chem. Phys. Lett. 142, 354 (1987).

[37] U. Bozkaya, J. M. Turney, Y. Yamaguchi, H. F. Schaefer, and C. D. Sherrill, J. Chem. Phys. 135, 104103 (2011).

[38] Horton 1.2.0 2013, written by T. Verstraelen, S. Vandenbrande, M. Chan, F. H. Zadeh, C. Gonzalez, K. Boguslawski, P. Tecmer, P. A. Limacher, A. Malek (see http://theochem.github.com/horton/).

[39] E. H. Lieb and F. Y. Wu, Phys. Rev. Lett. 20, 1445 (1968).

[40] E. Neuscamman, Phys. Rev. Lett. 109, 203001 (2012).

[41] L. Bytautas, T. M. Henderson, C. A. Jiménez-Hoyos, J. K. Ellis, and G. E. Scuseria, J. Chem. Phys. 135, 044119 (2011).

[42] H. Bethe, Z. Phys. 71, 205 (1931).

[43] E. H. Lieb and F. Y. Wu, Phys. Rev. Lett. 20, 1445 (1968).

[44] S.-J. Gu, S.-S. Deng, Y.-Q. Li, and H.-Q. Lin, Phys. Rev. Lett. 93, 086402 (2004).

[45] J. Hachmann, W. Cardoen, and G. K.-L. Chan, J. Chem. Phys. 125, 144101 (2006).

[46] T. Tsuchimochi and G. E. Scuseria, J. Chem. Phys. 131, 121102 (2009).

[47] L. Stella, C. Attaccalite, S. Sorella, and A. Rubio, Phys. Rev. B 84, 245117 (2011). 
[48] N. Lin, C. A. Marianetti, A. J. Millis, and D. R. Reichman, Phys. Rev. Lett. 106, 096402 (2011).

[49] W. J. Hehre, R. F. Stewart, and J. A. Pople, J. Chem. Phys. 51, 2657 (1969).
[50] J. P. Perdew, K. Burke, and M. Ernzerhof, Phys. Rev. Lett. 77, 3865 (1996). 